\title{
Improvement on Null Space LDA for Face Recognition: A Symmetry Consideration
}

\author{
Wangmeng Zuo ${ }^{1}$, Kuanquan Wang ${ }^{1}$, and David Zhang² \\ ${ }^{1}$ School of Computer Science and Technology, Harbin Institute of Technology, \\ Harbin, 150001, China \\ ${ }^{2}$ Biometrics Research Centre, Department of Computing, \\ The Hong Kong Polytechnic University, \\ Hung Hom, Kowloon, Hong Kong
}

\begin{abstract}
The approximate bilateral symmetry of human face has been explored to improve the recognition performance of some face recognition algorithms such as Linear Discriminant Analysis (LDA) and Direct-LDA (D-LDA). In this paper we summary the ways to generate virtual sample using facial symmetry, and investigate the three strategies of using facial symmetric information in the Null Space LDA (NLDA) framework. The results of our experiments indicate that, the use of facial symmetric information can further improve the recognition accuracy of conventional NLDA.
\end{abstract}

\section{Introduction}

It is well known that face has an approximate bilateral symmetry, which has been investigated in psychology and anthropology to study the relation of facial symmetry and facial attractiveness [1]. As to face recognition, Zhao et al. have utilized the facial symmetry to generate virtual mirrored training images [2]. More recently, the mirrored images are used as both training and gallery images [3]. Rather than the mirrored image, Marcel proposed another symmetric transform to generate virtual images [4].

Facial asymmetry also contains very important discriminative information for person identification. In [5], psychologists found the potential role of facial asymmetry in face recognition by humans. Recently, Liu revealed the efficacy of facial asymmetry in face recognition over expression variation [6]. Soon after they find that facial asymmetry can also be used to facial expression recognition.

While comparing with facial asymmetry, facial symmetry still has its advantageous properties. The measurement of facial asymmetry is based on the normalization of facial image according to the inner canthus $\left(\mathrm{C}_{1}, \mathrm{C}_{2}\right)$ of each eye and the philtrum $\left(\mathrm{C}_{3}\right)$. The accurate location of these three points, however, is practically very difficult due to the complexity of lighting and facial variation. Besides, the asymmetric discriminative information greatly suffers from the variation of lighting and pose. In [6], Liu investigate only the frontal face recognition problem. But for facial symmetry, it is natural to believe that face image has the symmetric illumination and pose variations.

Most current work on facial symmetry concentrates on two aspects, how to generate virtual images and how to use virtual images. For the first problem, Zhao pro- 
posed to generate the mirrored images [2] and Marcel proposed a symmetric transform to generate virtual samples [4]. For the second problem, most researchers use the LDA and Direct-LDA (D-LDA) frameworks [2, 3, 4].

In this paper, we extend to use facial symmetry in the Null Space LDA (NLDA) framework. Because of the particularity of NLDA, the common strategy to use symmetric information may be ineffective. We thus investigate some novel strategies, and comparatively evaluate them on two FERET face subsets.

\section{Null Space LDA: A Brief Review}

Null Space LDA (NLDA) is a natural extension of conventional LDA when the within-class scatter matrix $\mathbf{S}_{w}$ is singular [7, 8]. LDA intends to obtain the discriminant vector by maximizing the Fisher's linear discriminant criterion. When the within-class scatter matrix $\mathbf{S}_{w}$ is singular, we could find a subspace spanned by $\mathbf{U}=\left[\varphi_{1}, \varphi_{2}, \cdots, \varphi_{d}\right]$ (hereafter this subspace is named as the null space of $\mathbf{S}_{w}$ ) that satisfies

$$
\mathbf{U}^{T} \mathbf{S}_{b} \mathbf{U}>0 \text { and } \mathbf{U}^{T} \mathbf{S}_{w} \mathbf{U}=0 .
$$

where $\mathbf{S}_{b}$ is the between-class scatter matrix. In this subspace, the Fisher's discriminant criterion degenerates to

$$
J_{F L D}^{\prime}(\mathbf{w})=\left|\mathbf{w}^{T} \mathbf{U}^{T} \mathbf{S}_{b} \mathbf{U} \mathbf{w}\right|=\left|\mathbf{w}^{T} \tilde{\mathbf{S}}_{b} \mathbf{w}\right| .
$$

Another way to construct $\tilde{\mathbf{S}}_{b}$ is to find an orthonormal basis for the range of $\mathbf{S}_{w}$, $\mathbf{Q}=\left[\mathbf{u}_{1}, \mathbf{u}_{2}, \cdots, \mathbf{u}_{d_{\mathrm{w}}}\right]$. Then $\mathbf{S}_{b}$ can be projected into the null space of $\mathbf{S}_{w}$ by $\tilde{\mathbf{S}}_{b}=\mathbf{S}_{b}-\mathbf{Q}\left(\mathbf{Q}^{T} \mathbf{S}_{b} \mathbf{Q}\right) \mathbf{Q}^{T}$. The discriminant vectors of NLDA are obtained by calculating the eigenvectors of $\mathbf{U}^{T} \mathbf{S}_{b} \mathbf{U}$. By choosing the eigenvectors $\mathbf{W}=\left[\mathbf{w}_{1}, \mathbf{w}_{2}, \cdots, \mathbf{w}_{d_{\mathrm{NLDA}}}\right]$ corresponding to the first $d_{\mathrm{NLDA}}$ largest eigenvalues, we obtain the NLDA projector

$$
\mathbf{T}_{\mathrm{NLDA}}=\mathbf{U W} \text {. }
$$

From the previous discussion, the NLDA projector is easy to be calculated once we find the null or the range space of $\mathbf{S}_{w}$. Next, we review two methods in addressing this issue: by solving eigen-problems [7, 8] and by Gram-Schmidt orthogonalization [8].

\subsection{Constructing $\tilde{\mathbf{S}}_{b}$ by Solving Eigen-Problems}

To obtain the null space of $\mathbf{S}_{w}$, Yang proposed to first calculate all the eigenvectors $\boldsymbol{\Phi}=\left[\phi_{1}, \phi_{2}, \cdots, \phi_{d_{\mathrm{PCA}}}\right]$ corresponding to positive eigenvalues of the total scatter matrix $\mathbf{S}_{t}$. With the PCA projector $\boldsymbol{\Phi}$, we can construct a $d_{\mathrm{PCA}} \times d_{\mathrm{PCA}}$ matrix $\tilde{\mathbf{S}}_{w}$

$$
\tilde{\mathbf{S}}_{w}=\boldsymbol{\Phi}^{T} \mathbf{S}_{w} \boldsymbol{\Phi}
$$


Then, we calculate the eigenvectors corresponding to the zero eigenvalues of $\tilde{\mathbf{S}}_{w}$. Yang has proved that the subspace spanned by $\mathbf{V}=\left[\mathbf{v}_{d_{\mathrm{w}}+1}, \mathbf{v}_{d_{\mathrm{w}}+2}, \cdots, \mathbf{v}_{d_{\mathrm{PCA}}}\right]$ is the null space of $\tilde{\mathbf{S}}_{w}$ [7]. So we can obtain $\tilde{\mathbf{S}}_{b}=\mathbf{U}^{T} \mathbf{S}_{b} \mathbf{U}$, where $\mathbf{U}$ is defined as

$$
\mathbf{U}=\boldsymbol{\Phi V}=\left[\boldsymbol{\Phi} \mathbf{v}_{d_{\mathrm{w}}+1}, \boldsymbol{\Phi} \mathbf{v}_{d_{\mathrm{w}}+2}, \cdots, \boldsymbol{\Phi} \mathbf{v}_{d_{\mathrm{PCA}}}\right] .
$$

Actually, we can obtain $\tilde{\mathbf{S}}_{b}$ without calculating the eigenvectors of $\mathbf{S}_{t}$. In [8], Cevikalp proposed to compute the eigenvectors $\mathbf{Q}=\left[\mathbf{u}_{1}, \mathbf{u}_{2}, \cdots, \mathbf{u}_{d_{\mathrm{w}}}\right]$ corresponding to the positive eigenvalues of $\mathbf{S}_{w}$. Then $\mathbf{S}_{b}$ can be projected into the null space of $\mathbf{S}_{w}$ by

$$
\tilde{\mathbf{S}}_{b}=\mathbf{S}_{b}-\mathbf{Q}\left(\mathbf{Q}^{T} \mathbf{S}_{b} \mathbf{Q}\right) \mathbf{Q}^{T} .
$$

\subsection{Constructing $\tilde{\mathbf{S}}_{b}$ by Gram-Schmidt Orthogonalization}

Gram-Schmidt orthogonalization is introduced to speed up the computation of $\tilde{\mathbf{S}}_{b}$. Both the two methods in Section 2.1 require $\mathrm{O}\left(N^{3}\right)$ floating point multiplications. Actually all orthogonal basis for the range of $\mathbf{S}_{w}$ are equivalent. From this aspect, Cevikalp proposed a fast method with $\mathrm{O}\left(N^{2}\right)$ multiplications to constructing $\tilde{\mathbf{S}}_{b}$ [8].

Give a training set $\mathbf{X}=\left\{\mathbf{x}_{1}^{(1)}, \cdots, \mathbf{x}_{N_{1}}^{(1)}, \mathbf{x}_{1}^{(2)}, \cdots, \mathbf{x}_{j}^{(i)}, \cdots, \mathbf{x}_{N_{C}}^{(C)}\right\}$, we should first find the independent difference vector which spanned the difference subspace B. In [8], Cevikalp had proved the equivalence of the difference subspace and the range space of $\mathbf{S}_{w}$. Then Gram-Schmidt orthogonalization procedure is used to find an orthonormal basis $\mathbf{Q}=\left[\beta_{1}, \beta_{2}, \cdots, \beta_{N-C}\right]$ of $\mathbf{B}$. Next we can project the between-class scatter matrix $\mathbf{S}_{b}$ into the null space of $\mathbf{S}_{w}$ by $\tilde{\mathbf{S}}_{b}=\mathbf{S}_{b}-\mathbf{Q}\left(\mathbf{Q}^{T} \mathbf{S}_{b} \mathbf{Q}\right) \mathbf{Q}^{T}$.

\section{Strategies of Using Facial Symmetry in the NLDA Framework}

In this Section, we investigate the ways to utilize facial symmetry in the NLDA framework from two aspects. First, we summarize the ways to generate virtual images. Second, we investigate three possible methods to use facial symmetric transform.

\subsection{Two Ways to Generate Virtual Images Using Facial Symmetry}

We name the way to generate virtual images using facial symmetry as facial symmetric transform. So far, there are mainly two kinds of facial symmetric transform, SymmTrans-I and SymmTrans-II, defined as follows:

Definition 1. Given a facial image $\mathrm{A}=\left(a_{i, j}\right)_{m \times n}$, SymmTrans-I is defined to transform $\mathbf{A}$ to a new image $\mathbf{A}^{\prime}=\left(a_{i, j}^{\prime}\right)_{m \times n}$ by $a_{i, j}^{\prime}=a_{i,(n-j+1)}$.

Definition 2. Given a facial image $\mathrm{A}=\left(a_{i, j}\right)_{m \times n}$, SymmTrans-II is defined to transform A to a new image $\mathbf{A}^{\prime \prime}=\left(a_{i, j}^{\prime \prime}\right)_{m \times n}$ by $a_{i, j}^{\prime \prime}=\left(a_{i, j}+a_{i,(n-j+1)}\right) / 2$. 


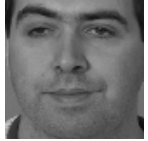

(a)

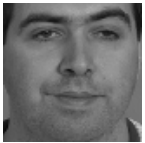

(b)

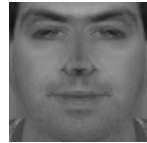

(c)

Fig. 1. Illustration of the results of facial symmetric transform: (a) original image; and the virtual images generated by (b) SymmTrans-I and (c) SymmTrans-II

These two facial symmetric transform had been reported in some literature. The virtual image generated by SymmTrans-I is usually called as mirrored image and has been applied in $[2,3]$. The virtual image generated by SymmTrans-II has been used in [4] and Marcel finds that SymmTrans-II can alleviate the effect of small pose variation. As an example, Fig. 1 illustrates the results of these two facial symmetric transform.

In NLDA, the image A should always be mapped to an image vector $\mathbf{a}$ in advance. Thus the virtual images generated by SymmTrans-I and SymmTrans-II should also be mapped to their corresponding image vectors, $\mathbf{a}^{\prime}$ and $\mathbf{a}^{\prime \prime}$.

\subsection{Three Methods to Use the Facial Symmetric Transform}

Facial symmetric transform can be used to generate virtual training images, virtual NLDA projector, or virtual gallery images. In this section, we investigate these three ways of utilizing facial symmetric information in the NLDA framework.

Generally, the NLDA-based face recognition system involves two stages, training and testing. In the training stage, the NLDA projector is obtained by learning from the training set and the gallery images are then projected into gallery feature vectors. In the testing stage, an image from the probe set is first projected into probe feature vector and then a nearest neighbor classifier is used to recognize the probe feature vector.

Fig. 2 4 illustrates the framework of using virtual training images, using virtual projector and virtual gallery images in NLDA-based face recognition. In Fig. 2, we use facial symmetric transform to obtain a virtual training set. Then both the training set and the virtual training set are used in the NLDA learning to obtain the projector (SymmNLDA-I). This is the most popular strategy of using facial symmetric transform and has been adopted in $[2,4]$. But for NLDA, this strategy may be ineffective because the addition of virtual training set may decrease the discriminative information in the null space of $\mathbf{S}_{w}$, and further degrade the recognition accuracy of NLDA.

In Fig. 3, facial symmetric transform is used to obtain a virtual projector. We use the NLDA projector and the virtual projector to extract two feature vectors, and then we combine the classification results based on these two feature vectors (Sym$m N L D A-I I)$. For details of the combination rule, see [9]. In Fig. 4, facial symmetric transform is used to obtain a virtual gallery set. Then both the gallery set and the virtual gallery set are used to construct the generalized gallery feature sets (Sym$m N L D A-I I I)$. 


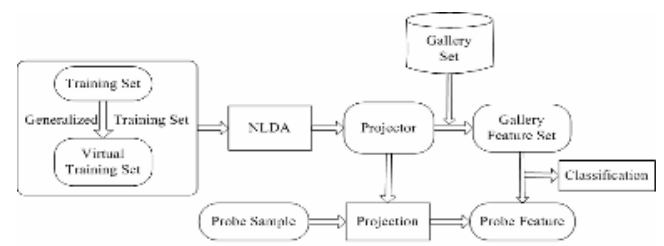

Fig. 2. An illustration of using virtual training set in the NLDA framework (SymmNLDA-I)

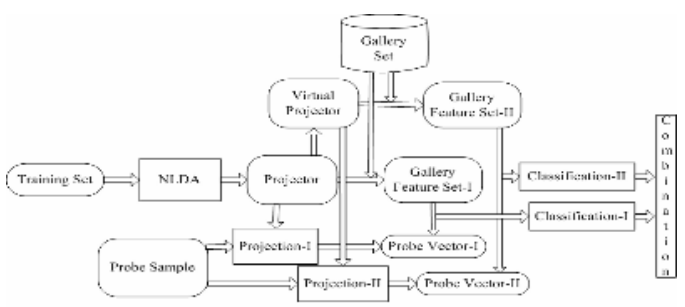

Fig. 3. An illustration of using virtual projector in the NLDA framework (SymmNLDA-II)

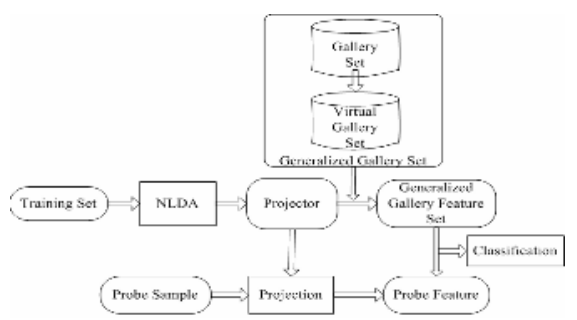

Fig. 4. An illustration of using virtual gallery set in the NLDA framework (SymmNLDA-III)

\section{Experimental Results and Discussions}

In this section, we use two face subsets from the FERET database (FERET-1 and FERET-2) to evaluate the facial symmetry in NLDA. To simplify the problem, we just compare the recognition rate of the three methods using SymmTrans-II.

\subsection{Experimental Results on FERET-1 Database}

In this section, we chose a subset from the FERET database (FERET-1) which includes 1,400 images of 200 individuals (each individual has seven images). The seven images of each individual consist of three front images and four profile images. The facial portion of each original image was cropped to a size of $80 \times 80$ and pre-processed using histogram equalization. Fig. 5 presents 7 cropped images of a person. 


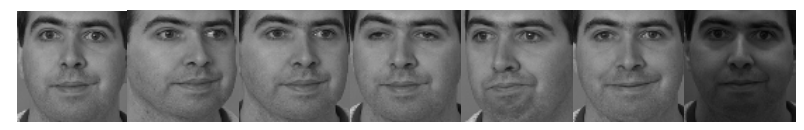

Fig. 5. Seven images of one person from the FERET-1 database

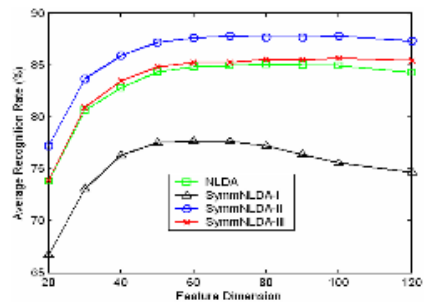

(a)

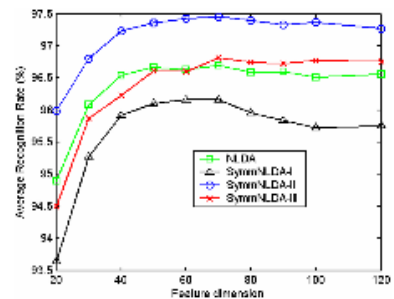

(b)

Fig. 6. Plots of the ARRs of NLDA, SymmNLDA-I, SymmNLDA-II, SymmNLDA-III: (a) FERET-1, and (b) FERET-2

The experimental setup is summarized as follows: First all the images of 100 persons are randomly selected for training. We use the 100 neutral frontal images of the other 100 persons as gallery images, and the remaining images as probe images. We run the recognition method 10 times to calculate the average recognition rate (ARR).

Fig. 6(a) depicts the ARRs obtained using NLDA, SymmNLDA-I, SymmNLDAII, and SymmNLDA-III. SymmNLDA-II and SymmNLDA-III achieve higher ARRs than NLDA and the highest ARR is obtained using SymmNLDA-II. But the ARR of SymmNLDA-I is much lower than that of NLDA, though the addition of virtual training samples has been reported to improve the recognition performance for subspace LDA and D-LDA $[2,4,3]$. NLDA extracts the discriminative information in the null space of $\mathbf{S}_{w}$. The addition of virtual training samples, however, enriches facial information in the range space of $\mathbf{S}_{w}$, and may degrade the recognition performance of NLDA.

\subsection{Experimental Results on FERET-2 Database}

We use a FERET subset consisted of 1195 people with two images (fa/fb) for each person (FERET-2). The facial portion of each image was cropped to a size of $80 \times 80$ and pre-processed by histogram equalization. Fig. 7 shows the ten pre-processed images of five persons. In our experiment, we randomly select 495 persons to construct the training set. Then, the 700 regular frontal images (fa) of the other 700 persons are used as gallery set, and the remained 700 images (fb) are used as probe set. We run the face recognition method 10 times and calculate the average recognition rate.

Fig. 6(b) illustrates the ARRs obtained using NLDA, SymmNLDA-I, SymmNLDA-II, and SymmNLDA-III. SymmNLDA-II and SymmNLDA-III also achieve higher maximum ARR than conventional NLDA and the highest ARR is obtained using SymmNLDA-II. But the ARR of SymmNLDA-I is lower than that of NLDA. 


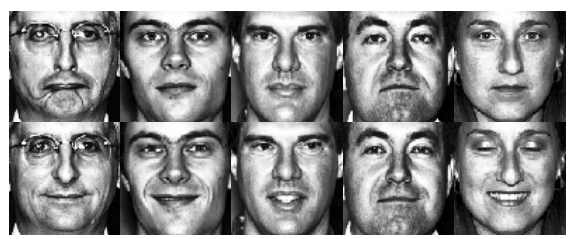

Fig. 7. Ten images of five persons from the FERET-2 database

\section{Conclusion}

In this paper we summary the facial symmetric transform (SymmTrans-I and SymmTrans-II) and the methods to use facial symmetry in the NLDA framework (SymmNLDA-I, SymmNLDA-II and SymmNLDA-III). Two face subsets from the FERET database are used to evaluate these methods. Experimental results show that SymmNLDA can further improve the recognition performance of NLDA. For a database of 1195 persons with expression variation, SymmNLDA-II achieves an average recognition rate of $97.46 \%$ with 495 persons for training and 700 persons for testing.

\section{Acknowledgements}

The work is partially supported by the NSFC fund under the contract No. 60332010 and No. 90209020.

\section{References}

1. Grammer, K., and Thornhill, R.: Human (Homo sapiens) facial attractiveness and selection: The role of symmetry and averageness. Journal of Comparative Psychology, 108 (1994) 233-242.

2. Zhao W, Chellappa R, Phillips P.J.: Subspace Linear Discriminant Analysis for Face Recognition. Tech Report CAR-TR-914, Center for Automation Research, University of Maryland (1999).

3. Lu, J., Plataniotis, K.N., and Venetsanopoulos, A.N.: Regularization studies of linear discriminant analysis in small sample size scenarios with application to face recognition. Pattern Recognition Letters, 26 (2005) 181-191.

4. Marcel, S., A symmetric transformation for LDA-based face verification. Proc. 6th IEEE Int'l Conf. Automatic Face and Gesture Recognition (2004) 207-212.

5. Troje, N.F., and Buelthoff, H.H.: How is bilateral symmetry of human faces used for recognition of novel views?. Vision Research, 38 (1998) 79-89.

6. Liu, Y., Schmidt, K.L., Cohn, J.F., and Mitra, S.: Facial asymmetry quantification for expression invariant human identification. CVIU, 91 (2003) 138-159.

7. Yang, J., Zhang, D., and Yang, J.Y.: A generalized K-L expansion method which can deal with Small Smaple Size and high-dimensional problems. PAA, 6(2003), 47-54.

8. Cevikalp, H., Neamtu, M., Wilkes, M., and Barkana, A.: Discriminative common vectors for face recognition. IEEE Trans. PAMI, 27(2005), 4-13.

9. Marcialis, G.L., Roli, F.: Fusion of appearance-based face recognition algorithms. Pattern Analysis and Applications, 7(2004), 151-163. 